Rev. Elev. Méd. vét. Pays trop., 1974, 27 (1) : 115-123

\title{
Evolution des incisives chez les zébus malgaches
}

\author{
par J. GILIBERT (*)
}

\begin{abstract}
RESUME
Utilisant une méthodologie mise au point par BRAZAL GARCIA, ROY et DUMONT, sur des Taurins, l'auteur a observé la dentition de 708 Zébus provenant de l'élevage extensif.

28 stades différents d'évolution individuelle d'une dent incisive et 35 degrés d'évolution de la mâchoire ont été distingués.

La comparaison avec des Taurins laitiers élevés en intensif en climat tempéré appelle les observations suivantes: si le remplacement des dents de lait par les dents d'adultes est à peu près synchrone, la sortie des dents est plus rapide chez le Zébu (pour les deux dentitions) et l'usure est plus lente (pour deux dentitions), indiquant une plus grande dureté des dents.

Les vaches Zébus étant mauvaises laitières et la quantité de veaux produite étant faible, on constate que la sortie rapide des dents et la longévité de la dentition sont une bonne adaptation aux conditions du milieu.
\end{abstract}

\section{INTRODUCTION}

L'évolution des incisives des Zébus et Taurins entretenus dans les conditions spéciales à l'élevage extensif en régions chaudes n'a, jusqu'ici, donné lieu qu'à des études fragmentaires et ponctuelles, d'où l'absence de codification. DUMAS et LHOSTE (3), par l'observation instantanée de 618 animaux d'âge connu, ont établi statistiquement l'âge moyen d'éruption des incisives chez des zébus métis Brahman $\mathbf{x}$ Foulbé élevés au Centre de Wakwa dans l'Adamaoua Camerounais.

BRAZAL GARCIA, ROY et DUMONT (1) ont défini des méthodes d'étude que l'on peut résumer de la façon suivante :

- distinction de 28 stades d'évolution pour une dent incisive;

- description de 35 degrés d'évolution de la

(*) Région de Recherches vétérinaires et zootechniques de Madagascar, B.P. 862, Tananarive, République Malgache. mâchoire par observation simultanée des stades d'évolution de chaque dent incisive;

- tracé d'une courbe de référence traduisant la relation du degré d'évolution de la mâchoire en fonction de l'âge.

Il a paru intéressant d'appliquer ces méthodes complètes et précises à l'étude de l'évolution de la dentition du Zébu malgache. Leur description détaillée ne sera pas reprise ici.

Les animaux sont tous des «Zébu Malgache $\gg$ élevés sur terrain sédimentaire, avec climat de type soudanien à saison sèche longue de 6 mois; 708 animaux ont été considérés.

Au cours du dépouillement, il n'est pas apparu de différences entre les sexes. Aussi les données ont-elles été regroupées pour la totalité des animaux.

Afin de simplifier le texte, les abréviations suivantes seront utilisées :

— pinces de lait . . . . . . $p$

- premières mitoyennes de lait . . $\mathrm{m}^{1}$

- deuxièmes mitoyennes de lait . . $\mathbf{m}^{2}$ 
- coins de lait . . . . . . c

- pinces adultes. . . . . . $\mathrm{P}$

- premières mitoyennes adultes . . $\mathbf{M}^{1}$

- deuxièmes mitoyennes adultes . . $\mathbf{M}^{2}$

- coins adultes. . . . . . C

\section{EVOLUTION INDIVIDUELLE D'UNE DENT INCISIVE}

Le tableau $\mathrm{n}^{\circ}$ I présente les 28 stades qui ont été différenciés et qui caractérisent l'évolution normale d'une incisive depuis l'apparition de la dent de lait jusqu'à la disparition de la dent d'adulte.

\section{Observations}

Il ne nous est pas apparu pour les dents adultes, à l'échelon individuel, un lien fidèle entre l'usure et l'apparition du collet puis de la racine; la synchronisation existe, bien sûr, puisqu'une dent qui n'est pas tout à fait à niveau présente une usure d'un millimètre environ et que la racine apparaît progressivement après l'arasement; cependant, pour les stades intermédiaires, les variations semblent très importantes.

La forme de la table d'usure est très généralement asymétrique par rapport au plan médian antéro-postérieur de la dent; la partie la plus proche de l'axe de la mâchoire s'use plus vite que la partie la plus éloignée; en conséquence, pour apprécier le degré de rasement de la table, il convient de faire une sorte de moyenne entre les deux côtés de la dent; le dessin du trèfle, formé par l'intersection du plan d'usure et de la face interne de la dent qui présente en son centre une petite éminence conique, l'avale, peut compliquer encore l'appréciation.

Pour les dents adultes, il apparaît que les quatre incisives se comportent un peu différemment : si le degré de rasement et l'état du trèfle sont synchrones, il n'en est pas de même pour l'évolution de la forme de l'étoile radicale qui est, à degré de rasement identique, un peu plus lente pour $\mathrm{M}^{2}$ et $\mathrm{C}$ que pour $\mathrm{P}$ et $\mathrm{M}^{1}$. La sortie de la dent de la gencive est moins avancée de $\mathrm{P}$ à $\mathrm{C}$ pour un même degré de rasement. De plus la concavité, critère difficile à apprécier au demeurant, diminue de $\mathrm{P}$ à $\mathrm{C}$ pour un même degré de rasement.

Pour les dents de lait comparées aux dents d'adultes, il apparaît que pour une usure identique, la sortie et le déchaussement de la dent, ainsi que l'évolution de la forme de l'étoile radicale (souvent triangulaire d'ailleurs) étaient plus précoces. Par contre, la concavité de la table d'usure est sensiblement moins accentuée.

Pour l'usure, quelques anomalies ont été observées :

- Chez certains animaux, les huit dents adultes sont plantées, non dans le prolongement du maxillaire, mais selon un angle se rapprochant de la perpendiculaire. L'usure ne se produit pas alors de la façon normale; par exemple la racine commence à être usée sur l'avant alors que la dent n'est pas totalement rasée sur l'arrière;

- A partir du stade XX, on a observé quelquefois que les pinces étaient usées en biseau, la partie proche du plan antéro-postérieur de la mâchoire s'usant plus vite; la forme de la table d'usure et celle de l'étoile radicale sont considérablement modifiées.

Sur un plan plus général, les anomalies suivantes ont été observées:

- Les coins adultes peuvent ne pas apparaître, ce fut le cas pour 12 animaux de plus de six ans sur 285;

- Les dents d'adultes sont quelquefois en position anormale, par un retournement de 90 à 180 degrés autour de l'axe de la dent. Treize mâchoires ont présenté ce phénomène, jamais vu sur les pinces mais sept fois sur les $\mathrm{M}^{1}$ (dont quatre fois symétriquement), deux fois sur les $\mathrm{M}^{2}$ et quatre fois sur les coins (dont une fois symétriquement). L'usure de ces dents est bien entendu aberrante;

- Des caries sur l'ivoire de néoformation, intervenant généralement après le rasement de la dent ont été observées sur 21 animaux, dont trois caries doubles et une carie quadruple. Ces caries ne gênent probablement pas l'animal.

\section{Comparaison des Taurins laitiers en climat tempéré et élevage intensif}

La comparaison du tableau $\mathbf{n}^{\circ}$ I avec le tableau correspondant de BRAZAL et collab. (1) fait apparaître les différences suivantes :

- Pour les dents de lait, l'apparition du collet puis celle de la racine sont plus précoces chez le zébu malgache; 


\section{Tableau $n^{0} 1$}

Evolution individuelie d'une dent inclsive

\begin{tabular}{|c|c|}
\hline Stade 0 & Ia dent de lait n'tat pas sortie. \\
\hline Stade I & $\begin{array}{l}\text { La dent, sortie, est d'implantation irrégulière. Ulobile, elle se chevauche } \\
\text { avec les autres. }\end{array}$ \\
\hline Stade II & $\begin{array}{l}\text { La dent est 1mplantée plus solidement. La position se régularige, mais elle } \\
\text { chevauche encore un peu avec sey voisines. }\end{array}$ \\
\hline Stade III & $\begin{array}{l}\text { La dent, réguliérenent plantée, n'est en contact avec ses volsines que par } \\
\text { les bords latéraux. }\end{array}$ \\
\hline Stade IV & La dent a terminé sa crolssance, elle est à niveau. \\
\hline Stade V & Ragement do la dent marqué par une légère usure du bord supérieur. \\
\hline Stade VI & Le collet est bien dégagé de la gencive. \\
\hline Stade VII & La racine apparaît. Le dent est nivelée à moitié. \\
\hline Stade VIII & $\begin{array}{l}\text { La dent est presque complètement ntvelée, le trèfle e日t net; la recine } \\
\text { est blen visible. }\end{array}$ \\
\hline Stade IX & $\begin{array}{l}\text { La dent est complètement nivelée. Le trèfle a disparu. La racine est trèa } \\
\text { déchaussée. }\end{array}$ \\
\hline Stade $X$ & La dent bouge. \\
\hline Stade $X I$ & La dent de lait est tombée. ; la dent d'adulte apparaft. \\
\hline Stade $X I I$ & La dent adulte est entre 0 et $3 / 4$ du niveau \\
\hline Stade XIII & $\begin{array}{l}\text { La dent adulte dépasse les } 3 / 4 \text { du niveau, elle attelnt un premier degré de } \\
\text { rasement de } 1 / 2 \text { à } 1 \text { ma. }\end{array}$ \\
\hline Stade XIV & 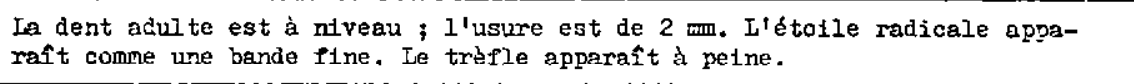 \\
\hline Stade XV & $\begin{array}{l}\text { La dent atteint un } 3 \mathrm{e} \text { stade de rasement de } 3 \mathrm{~mm} \text {. L'étoile reste une bande } \\
\text { fine. Le trèfle est très net, la concevité de la surface d'usure est sensible }\end{array}$ \\
\hline Stade XVI & $\begin{array}{l}\text { Le rasement intéresse le } 1 / 3 \text { supérieur de la dent. L'étolle radicale est } \\
\text { encore une bande. Le collet apparait. }\end{array}$ \\
\hline Stade XVII & $\begin{array}{l}\text { Ire rasement intéresse la mojtlé de la dent. I'étoile commence à devenir un } \\
\text { rectangle allongé. Le collet est net. Le trèfle est net. }\end{array}$ \\
\hline Stade XVIII & $\begin{array}{l}\text { Ie rasement intéresse les } 3 / 4 \text { de la dent. I'étolle comence à devenir un } \\
\text { rectangle plus ou molins allongé. Le trèfle cormence à g'estomper. }\end{array}$ \\
\hline Stade $X I X$ & $\begin{array}{l}\text { Ia dent est nivelée : I'étoile radicale rectangulaire se rapproche du centre. } \\
\text { Le trèfle a disparu. Ia concavité s'affirme. }\end{array}$ \\
\hline Stade $X X$ & $\begin{array}{l}\text { I'étoile radicale devient un rectazgle large. Ia forte de la table eat tra- } \\
\text { pézoíale. ia racino de la dent apparat. }\end{array}$ \\
\hline Stade XXI & I'étolle radicale est un carré. Ia table est un trapéze aux argles arrondis. \\
\hline Stade XXI & $\begin{array}{l}\text { L'étolle radicale e日t un carré aux angles furrondis ; la table devient ronde ; } \\
\text { l'usure atteint le volsinage du collet. }\end{array}$ \\
\hline Stode $x I I I$ & $\begin{array}{l}\text { L'stoxle radicule est ronde. In table est très concave. La racine est dé- } \\
\text { chaussée. }\end{array}$ \\
\hline Stadc XXIV & Io dent est très raccourcle par l'usure; elle bouge. \\
\hline Stade XXV & $\begin{array}{l}\text { Ia dent n'est plus reprécentéo que par le racine; elle est très écartée de } \\
\text { ses voisines }\end{array}$ \\
\hline Stade XXYI & In dent n'est plus qu'un bout de racine (cliteot). Flle peut Etre tombee. \\
\hline Stade XXYII & Touts \\
\hline
\end{tabular}


Tobleau $n^{\circ}$ U

Degrés d'évalution de le dent1tion

\begin{tabular}{|c|c|c|c|c|c|c|c|c|c|}
\hline \multicolumn{10}{|c|}{ Taurins } \\
\hline $\begin{array}{c}\text { Degréce } \\
\text { dievolution }\end{array}$ & $P_{1}$ & $\mathrm{H}_{1} \mathrm{I}$ & $\mathrm{M}_{2} \mathrm{I}$ & $\mathrm{c}$ & $P A$ & $\mathrm{~B}_{1} \mathrm{~A}$ & $\mathrm{Br}_{2} \mathrm{~A}$ & or & Age \\
\hline 0 & $I$ & I & & & & & & & $0-30$ jours \\
\hline 1 & I & I & I & I & & & & & 1 nots \\
\hline 2 & II & II & $I$ & $I$ & & & & & 45 jouts \\
\hline 3 & III & III & II & II & & & & & 3 mois \\
\hline 4 & IV & IV & $I V$ & IV & & & & & 5 mols \\
\hline 5 & $\gamma$ & $v$ & Ir & IV & & & & & 6 nols \\
\hline 6 & $\mathrm{v}$ & $\mathrm{v}$ & $r$ & $I V, V$ & & & & & 9 mols \\
\hline 7 & $V I$ & $\mathrm{vI}$ & $\mathrm{v}$ & $\mathrm{v}$ & & & & & 11 mois \\
\hline 8 & VII & $\overrightarrow{\text { VII }}$ & $\overrightarrow{7}$ & $\mathrm{r}$ & & & & & 12 mo1s \\
\hline 9 & $\begin{array}{r}\text { VIII } \\
\text { IX } \\
\end{array}$ & VIII & $\mathrm{v}$ & v & & & & & 14 mo1s \\
\hline 10 & $I X$ & $I X$ & VI & $\mathrm{v}$ & & & & & 15 mois \\
\hline 11 & $I X$ & $I X$ & $I X$ & $i$ & & & & & 16 noia \\
\hline 12 & $I X$ & $I X$ & $I X$ & VI & & & & & 18 mois \\
\hline 13 & $I X$ & $I X$ & $I X$ & $I X$ & & & & & 20 mods \\
\hline 14 & $x$ & $I X$ & $I X$ & $I X$ & & & & & 22 mo1s \\
\hline 15 & & $i x$ & $I X$ & $I Y$ & XI & & & & 24 mois \\
\hline 16 & & & $I x$ & $I X$ & XII & $x I$ & & & 30 mojs \\
\hline 17 & & & $x$ & $I K$ & $x I I$ & XII & & & 36 mo1s \\
\hline 18 & & & & $I X$ & XIII & XII & $\mathrm{XI}$ & & 42 n101s \\
\hline 19 & & & & $I X$ & XIV & YIII & $K I I$ & & 48 mols \\
\hline 20 & & & & $x$ & $x v$ & $X I V$ & XIII & & 52 mois \\
\hline 21 & & & & & $x y$ & $\mathrm{XIV}$ & XXII & $x I$ & 56 mois \\
\hline 22 & & & & & XVI & $\mathrm{XY}$ & $\mathrm{XIV}$ & $\mathrm{xI}$ & 60 mois \\
\hline 23 & & & & & $x y$ & $x y$ & $x I V$ & XII & 56 moss \\
\hline 24 & & & & & XrI & $\mathrm{xv}$ & $x I V$ & XIII & 72 mons \\
\hline 25 & & & & & XYII & $\mathrm{xN}$ & $x V$ & $x I V$ & 73 nno1s \\
\hline 26 & & & & & XYIII & XVII & $x V$ & $\mathrm{xN}$ & B4 tois \\
\hline 27 & & & & & $x I X$ & XVIII & XVII & $x V$ & 90 mots \\
\hline 28 & & & & & $\mathrm{xx}$ & $x I x$ & XVIII & WVI & $8 \mathrm{nns}$ \\
\hline 29 & & & & & $x x x$ & $x x$ & $X I X$ & XVIII & $2 \mathrm{ang}$ \\
\hline 30 & & & & & $x \times I I$ & $x$ & $x x$ & $x x$ & $10 \mathrm{cns}$ \\
\hline 31 & & & & & $X X I I \bar{I}$ & $x \in I \overline{1}$ & $\mathrm{XKI}$ & $\mathrm{xx}$ & 11 ans \\
\hline 32 & & & & & yKIY & axIII & $X: I I$ & $\mathrm{XXI}$ & $12 \mathrm{nns}$ \\
\hline 33 & & & & & $x, y$ & XYIV & XXIII & WEII & 13 ans \\
\hline 31 & & & & & $x v_{I}$ & $\cdot w$ & Y:XIV & NIII & 14 Enis \\
\hline
\end{tabular}


Tabzea: II $^{\circ}$ II

Degrés d'évolution de la dentition

\begin{tabular}{|c|c|c|c|c|c|c|c|c|c|}
\hline $\begin{array}{c}\text { Degrés } \\
\text { d' évolution }\end{array}$ & $p_{1}$ & $h_{1} 1$ & $\mathrm{BH}_{2} \mathrm{I}$ & $c_{1}$ & PA & $\mathrm{M}_{1} \mathrm{~A}$ & $\mathrm{H}_{2}^{\mathrm{A}}$ & $\mathrm{CA}$ & Age \\
\hline 0 & I & I & & & & & & & $0-5$ jours \\
\hline 1 & II & II & $I$ & $I$ & & & & & 10 jours \\
\hline 2 & III & II & I & I & & & & & 15 fours \\
\hline 3 & IV & III & II & $I I$ & & & & & 1 mots \\
\hline 4 & $\nabla$ & IV & III & II & & & & & $2 \mathrm{mols}$ \\
\hline 5 & $v$ & $\mathrm{Y}$ & IV & IV & & & & & 3 m.01s \\
\hline 6 & $V I$ & $v$ & IV & IV & & & & & $4 \operatorname{mots}$ \\
\hline 7 & VI & $V I$ & V & IV & & & & & $6 \mathrm{~m} .01 \mathrm{~s}$ \\
\hline 8 & vI & VI & $\mathrm{VI}$ & $\mathrm{v}$ & & & & & 9 5019 \\
\hline 9 & VT & VI & VI & VI & & & & & 11 бa:a1s \\
\hline 10 & VII & $\mathrm{VI}$ & VI & VI & & & & & 15 mols \\
\hline 11 & VII & VII & $\mathrm{VI}$ & VI & & & & & 16 mods \\
\hline 12 & VIII & VII & VI & VI & & & & & 18 mois \\
\hline 13 & $I X$ & VII & $\mathrm{VI}$ & VI & & & & & 20 घ.ois \\
\hline 14 & IX & VIII & VII & $V I$ & & & & & 22 mo1s \\
\hline 15 & & VIII & VII & $\Psi I$ & $X I$ & & & & 25 mois \\
\hline 16 & & VIII & VII & VI & SII & & & & $29 \mathrm{mols}$ \\
\hline 17 & & & VIII & VII & $x I V$ & $\mathrm{XI}$ & & & 33 mois \\
\hline 18 & & & $x$ & VIII & XI & $x \Gamma$ & & & 40 m.ois \\
\hline 19 & & & & $I X$ & $X V I$ & $x v$ & $x I$ & & 45 mo1s \\
\hline 20 & & & & $x$ & $X V I$ & $X V I$ & XIII & & 48 drois \\
\hline 21 & & & & & $x V I$ & $X V I$ & $\mathrm{xIV}$ & $x I$ & 52 mons \\
\hline 22 & & & & & YVII & $x w I$ & $X V I$ & xIII & 58 mois \\
\hline 23 & & & & & XVII & XVI & $X V I$ & XIII & $64 \mathrm{mozs}$ \\
\hline 24 & & & & & WIII & XVII & $X V I$ & $x V$ & 70 m02. \\
\hline 25 & & & & & XVIII & WIII & $X V I$ & $X V I$ & 79 mo1s \\
\hline 26 & & & & & $x I X$ & YVIII & XVII & XVI & 86 mors \\
\hline 27 & & & & & 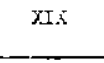 & $\begin{array}{r}X I I I I \\
\text { et } X I X \\
\end{array}$ & XVII & $x V I$ & 8 ans \\
\hline 28 & & & & & $\sigma i$ & iIX & AIII & AVII & $\theta$ ans \\
\hline 29 & & & & & $x X I$ & XI i & XVIII & XVII & $9 \mathrm{cns}$ \\
\hline 30 & & & & & XIII & $x x$ & $x I X$ & DVIII & $10 \mathrm{~ns}$ \\
\hline 31 & & & & & MII & $x X I$ & $\ddot{x}$ & XVIII & 31 ans \\
\hline 32 & & & & & $\operatorname{aIv}$ & $X \times I I$ & $\ddot{Z} X I$ & ZVIII & 12 pans \\
\hline 33 & & & & & $x \vee$ & XXIII & $\because \mathrm{II}$ & $\therefore \ddot{x}$ & 13 ans \\
\hline 34 & & & & & XVII & $Y \% N$ & ASIII & $x x$ & 14 en: \\
\hline
\end{tabular}


- La dent adulte commence à être usée avant de terminer sa croissance;

- A un degré de rasement donné, la forme de l'étoile radicale est en retard chez le Zébu par rapport au Taurin;

- Le collet apparaît plus tôt (stade XVI au lieu de XVII), de même que la racine (stade XX au lieu de XII);

- Le trèfle se maintient plus longtemps pour un même degré de rasement;

- Le temps nécessaire pour que l'usure atteigne le voisinage du collet est plus long chez le Zébu (stade XXII au lieu de XX);

- La concavité semble apparaître plus tôt chez le Zébu (stade XV au lieu de XVII).

En raison des différences qui viennent d'être énumérées, la définition des différents stades d'évolution d'une dent n'a pu être identique à celle des Taurins. Nous nous sommes cependant efforcés d'affecter le même numéro de stade d'évolution aux critères les plus importants (apparition de la dent, degré de rasement, déchaussement...) afin que les comparaisons soient possibles.

\section{DEGRE D'EVOLUTION DE LA DENTITION}

Le tableau $\mathbf{n}^{\circ}$ II présente 35 degrés d'évolution de la mâchoire de la naissance à 14 ans.

\section{Observations}

Un degré d'évolution caractérise les stades d'évolution simultanée des quatre incisives; une certaine variabilité existe parmi les animaux; au tableau $\mathrm{n}^{\circ}$ II, les quatre stades correspondant à un degré d'évolution sont l'appariement le plus fréquent.

L'âge auquel les animaux présentent un degré d'évolution donné manifeste une variabilité assez grande comme il avait été observé dans une étude précédente. En conséquence, au tableau $\mathrm{n}^{*}$ II, l'âge affecté à un degré d'évolution donné traduit bien entendu une moyenne.

\section{Comparaison avec les Taurins laitiers de climat tempéré}

\section{- Dents de lait}

La sortie et la croissance des dents de lait est beaucoup plus précoce chez le Zébu Mal- gache que chez les Taurins, comme le montre le tableau $\mathrm{n}^{\circ}$ III.

Mais, à partir de l'âge d'un an environ, la différence s'estompe et les Taurins vont atteindre les stades d'usure plus précocement que les Zébus, comme on le voit dans le tableau IV.

La nourriture consommée par les Zébus étant certainement plus grossière que celle des Taurins, on peut penser que les dents des premiers sont plus dures que celles des seconds.

\section{- Apparition des dents adultes}

Les quatre dents adultes apparaissent à 25 mois, 33 mois, 45 mois et 52 mois chez le Zébu, contre 24 mois, 30 mois, 42 mois et 56 mois respectivement chez les Taurins; les Zébus sont donc légèrement en retard pour les trois premières ( -1 mois, -3 mois, - 3 mois) et en avance pour les coins ( +4 mois); on ne peut conclure à une précocité nette d'une race par rapport à l'autre pour ce critère.

\section{Remarque}

Dans une autre série d'observations, les mêmes animaux Zébu malgache ont été examinés à âges fixes pendant six ans, sur l'existence ou non des dents adultes. L'âge moyen d'apparition des dents adultes a été calculé en prenant la moyenne des deux extrêmes observés, selon l'exemple suivant: l'âge théorique de la sortie de $\mathrm{M}^{1}$ était la moyenne entre le plus jeune animal observé avc $\mathbf{M}^{1}$ adulte et le plus vieil animal observé avec seulement les $\mathbf{P}$ adultes (tableau $\mathrm{n}^{0} \mathrm{~V}$ ). Les résultats concordent remarquablement selon les âges indiqués (tableaux $\mathrm{n}^{\circ} \mathrm{V}$ et $\mathrm{VI}$ ).

\section{- Evolution des dents adultes}

De même que pour les dents de lait, la poussée des dents d'adultes est beaucoup plus rapide chez les Zébus que chez les Taurins; entre le stade XI où la dent apparaît et le stade XIV où elle est à niveau, les temps écoulés sont notés au tableau $\mathrm{n}^{\circ}$ VII.

En conséquence, l'usure des dents adultes accuse une avance chronologique importante chez les Zébus, dans les premières années de la dentition adulte; ainsi le stade XVI, pour lequel le rasement intéresse le tiers supérieur de la dent, est atteint aux âges indiqués au tableau $\mathrm{n}^{\mathrm{o}}$ VIII.

L'usure des dents est ensuite beaucoup plus rapide chez les Taurins, comme on peut le voir 


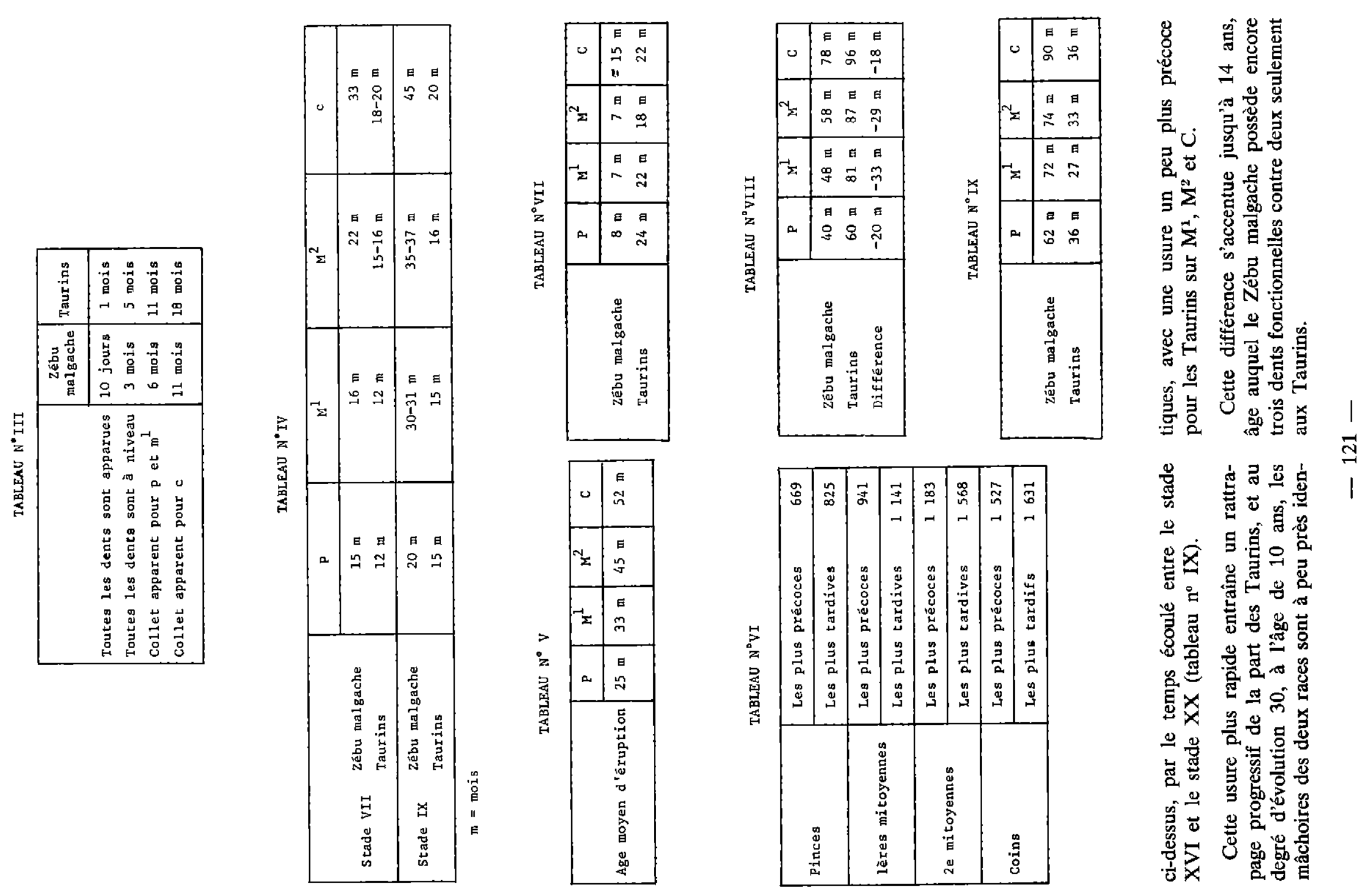




\section{CONCLUSION}

La méthode très précise mise au point par BRAZAL GARCIA, ROY et DUMONT sur des Taurins a pu être utilisée sur le Zébu; quelques modifications dans les critères de définition de quelques stades d'évolution d'une dent incisive sont apparues indispensables.

Il faut préciser que les résultats obtenus concernent une race de Zébus, élevés dans des conditions écologiques données. D'autres races élevées dans d'autres conditions présenteraient peut-être des résultats sensiblement différents.

La comparaison des degrés d'évolution de la dentition montre très clairement que les dents sortent plus rapidement et sont à niveau plus précocement chez les Zébus que chez les Taurins, aussi bien pour les dents de lait que pour les dents d'adultes. L'usure de la dent est par contre nettement plus rapide chez les Taurins que chez les Zébus, aussi bien pour les dents de lait que pour les dents d'adultes; comme la nourriture consommée par les Taurins d'élevage intensif est certainement plus tendre que celle des Zébus d'élevage extensif, l'hypothèse la plus vraisemblable paraît être une dureté plus grande des dents des Zébus.
Le remplacement des dents de lait est un peu plus précoce chez les Taurins pour les pinces et les deux mitoyennes, plus tardif pour les coins; on ne peut donc conclure à une précocité supérieure des Taurins, comme on aurait pu l'attendre de la part de races améliorées.

Les conséquences pratiques d'une sortie plus rapide de dents plus dures sont les suivantes :

- Le Zébu est apte plus tôt à consommer une nourriture grossière (dès l'âge d'un mois, il pâture effectivement);

- La dentition des Taurins est ruiniforme à 14 ans alors que celle des Zébus est encore fonctionnelle à cet âge pour plusieurs années, entraînant une longévité pratique plus grande (des vaches productives de 20 ans ne sont pas rares).

Si l'on sait, par ailleurs que, d'une part, les vaches Zébu sont mauvaises laitières et que, d'autre part, la quantité de veaux produite est faible, on constate que la sortie rapide des dents et leur usure relativement lente sont une bonne adaptation aux conditions du milieu. Elles permettent une alimentation solide plus précoce des jeunes et la compensation d'une faible fécondité, par une plus grande longévité des femelles reproductrices.

\section{SUMMARY}

\section{Incisor evolution in malagasy Zebu cattle}

Using a methodology restated by BRAZAL GARCIA, ROY, DU. MONT, with Bos taurus, the author has studied the dentition of 708 Bos indicus coming from extensive breeding.

28 different stages of individual evolution of an incisor and thirty five degrees of evolution of the jaw have been distinguished.

The comparison with milk Bos taurus in intensive breeding under temperate climate needs the following comments : although the substitution of the milk dentition by the permanent teeth is nearly synchronous, the growth of teeth is swifter for zebu (for the two dentitions) and the attrition is slower (for the two dentitions), indicating that teeth are more tough.

Zebu cows giving few milk and fecondity being small, we establish that the swift growth of teeth and the longevity of the dentition show a good adaptation to the general conditions.

\section{RESUMEN}

Evolución de los incisivos en los cebues de Madagascar

Utilizando una metodologia puesta a punto por BRAZAL GARCIA, ROY, DUMONT, sobre Bos taurus, el autor observó la dentición de 708 cebues proviniendo de la cria extensiva.

Se notaron 28 estados diferentes de evolución individual de una diente incisiva y 35 grados de evolución de la quijada.

Según la comparación con Bos taurus lecheros criados intensivamente 
bajo clima templado se observa que : si la sustitución de los dientes de leche por los dientes de adultos es poco más o menos sincrónica, el crecimiento de los dientes es más rapido en el cebú (en cuanto a las dos denticiones) y el desgaste es más lento (en cuanto a las dos denticiones), indicando una dureza más grande.

Siendo las vacas cebù malas lecheras y la cantidad de terneros producida poco importante, se comproba que el crecimiento rápido de los dientes y la longevidad de la dentición representan una buena adaptación a las condiciones del medio.

\section{BIBLIOGRAPHE}

1. BRAZAL GARCIA (T.), ROY (G.), DUMONT (B. L.). Evolution des incisives chez les bovins. Rev. Elev. Méd. vét. Pays trop, 1971, 24 (1): 53.68.

2. CORNEVIN (C.), LESBRE (X.). Traité de l'âge des animaux domestiques. Paris, Baillère et fils, 1894.
3. DUMAS (R.), LHOSTE (P.). Les signes de l'âge chez le Zébu. Etude des incisives de remplacement. Rev. Elev. Méd. vét. Pays trop, 1966, 19 (3) : 357-363.

4. METZGER (G.). Contribution à l'étude anato. mique des incisives et de l'âge du Zébu de Madagascar. Thèse, Méd., vét. Alfort. 1933, no 32, 54 p. 